\title{
Standards of Reporting-Another Hurdle to Get a Manuscript Accepted?
}

\author{
Klaus Hausegger ${ }^{1}$
}

Published online: 8 February 2022

(C) Springer Science+Business Media, LLC, part of Springer Nature and the Cardiovascular and Interventional Radiological Society of Europe (CIRSE) 2022

Recently the publisher of "Cardiovascular and Interventional Radiology" Springer, sent a policy update for reporting standards of biomedical research submitted for possible publication in their portfolio of journals. In this letter the publisher requests a checklist is completed for manuscripts of Randomized controlled trials, randomized controlled trial protocols, systematic reviews and meta-analysis, case reports and preclinical animal studies, which can be found online at "https:// www.equator-network.org.". In addition, the publisher strongly recommends that authors adhere to the STROBE guidelines for observational studies and several other study types.

Currently we are living in an environment of increasing regulations, certifications and control. This trend may be seen to be counterproductive for academic research and writing, compromising the principles of freedom of research. Increasing regulations may also be seen to potentially stifle innovation in science and the generation of new and novel ideas.

However, we have to consider what is the purpose of scientific writing and publishing? This is to accurately communicate the results of research in a coherent manner to make it as clear and easy to follow as possible to readers. The results have to be factual and should be reported in as concise a manner as possible with a balanced interpretation. This process is very much comparable to the writing of a radiological report. Such a report should contain a concise description of the findings followed by an interpretations of these findings. It has been well documented that structured reporting is a very useful tool to help improve the quality of radiological reports (lit.), particularly for younger and non, native speaking radiologists.

Many manuscripts submitted to CVIR are often on topics which are novel or very interesting suffer from poor methodology and structure. Consequently, a large proportion of our editorial work consists of sending recommendations on how to improve the structure of manuscripts so that they may be considered for publication.

Following a standardized guideline for writing manuscripts form the outset of writing can be a big help. It is a win for both the authors and reviewers as they can be sure that all necessary issues are addressed from the start making it easier to understand and thus the paper more likely to be well received by the scientific community. If a reviewer is confronted with a poorly structured paper, it makes the review process more difficult and slower. This will usually result in a more critical and negative review resulting in a greater likelihood of rejection.

For all these reasons it is clear that specific guidelines can be very helpful to the scientific community and should be followed in scientific writing. The most widely accepted guidelines can be can be found on the Equator Network website. Should the authors submit a completed checklist together with their manuscript? From our perspective it is a clear "Yes". Current instructions to authors for CVIR already mandate adherence to STROBE guidelines which will now be extended to

\footnotetext{
Klaus Hausegger

CVIR.hausegger@kabeg.at
}

1 Department of Diagnostic and Interventional Radiology, Klagenfurt State Hospital, Klagenfurt, Austria 
other manuscript types. What happens if no check list is provided? Well, we will have to go back to the authors to submit this as required by the publisher which will inevitably delay the manuscript handling process.

Coming back to the original question "Standards of reporting-another hurdle to get a manuscript accepted?" the clear answer is "No it is not". Rather than hinder research reporting, it will actually improve and accelerate the process of dissemination of knowledge to the scientific community.

Therefore, please visit the EQUATOR Network homepage and download the respective checklists, fill in these checklists properly and submit it together with your manuscript.

Funding None.

Declarations

Conflict of interest There is no conflict of interest.

Publisher's Note Springer Nature remains neutral with regard to jurisdictional claims in published maps and institutional affiliations. 